\title{
Risk Factors for the Presence of Symptoms in Peptic Ulcer Disease
}

\author{
Sang Pyo Lee, In-Kyung Sung, Jeong Hwan Kim, Sun-Young Lee, Hyung Seok Park and Chan Sup Shim \\ Department of Internal Medicine, Konkuk University School of Medicine, Seoul, Korea
}

Background/Aims: Peptic ulcer disease (PUD) is a common condition, but is difficult to detect in asymptomatic individuals. We aimed to investigate the prevalence of symptomatic and asymptomatic PUD during screening endoscopy and to identify risk factors for the presence of symptoms in patients with PUD.

Methods: We investigated subjects who underwent a health inspection, including endoscopy of the upper gastrointestinal (GI) tract and a serum anti-Helicobacter pylori IgG assay, and who completed a self-report questionnaire about their symptoms.

Results: Of the 12,852 subjects included in the study, 124 (1.0\%) had symptomatic PUD and 309 (2.4\%) had asymptomatic PUD. Old age, current smoking, and $H$. pylori infection were independent risk factors for symptomatic and asymptomatic PUD. Use of nonsteroidal anti-inflammatory drugs (NSAIDs) was an independent risk factor only for symptomatic PUD ( $p=0.040)$. Compared to subjects with asymptomatic PUD, subjects with symptomatic PUD were more likely to have active-stage ulcers $(p=0.002)$ and to drink more heavily $(p=0.005)$.

Conclusions: Use of NSAIDs is a risk factor for symptomatic PUD, but not for asymptomatic PUD. Excessive alcohol consumption and activestage ulcers in patients with PUD are related to the presence of gastroduodenal symptoms. Clin Endosc 2017;50:578-584

Key Words: Peptic ulcer; Anti-inflammatory agents, non-steroidal; Helicobacter pylori; Heavy drinking

\section{INTRODUCTION}

Peptic ulcer disease (PUD) is a common disease and results in various complications such as bleeding, perforation, and gastric outlet obstruction. ${ }^{1,2}$ Helicobacter pylori infection and the use of non-steroidal anti-inflammatory drugs (NSAIDs) are the most well-known causal factors for PUD. ${ }^{3-7}$ Although the prevalence of PUD caused by $H$. pylori has been decreasing because of eradication therapy, the prevalence of PUD induced by NSAIDs or aspirin is increasing because of the worldwide increase in the aging population. ${ }^{8-10}$ In areas of endemic $H$. pylori infection such as Korea, the prevalence rate

Received: September 2, 2016 Revised: October 24, 2016

Accepted: October 31, 2016

Correspondence: In-Kyung Sung

Department of Internal Medicine, Digestive Disease Center, Konkuk University School of Medicine, 120-1 Neungdong-ro, Gwangjin-gu, Seoul 05030, Korea Tel: +82-2-2030-5100, Fax: +82-2-2030-7748, E-mail: inksung@kuh.ac.kr

(cc) This is an Open Access article distributed under the terms of the Creative Commons Attribution Non-Commercial License (http://creativecommons.org/ licenses/by-nc/3.0) which permits unrestricted non-commercial use, distribution, and reproduction in any medium, provided the original work is properly cited. of $H$. pylori infection is as high as $66.9 \%$, and people in this region may be at high risk for PUD. Therefore, new strategies for the prevention and cure of PUD in Korea are important. ${ }^{11}$

It is difficult to detect PUD in asymptomatic individuals. In some cases, it is detected because of serious complications, whereas in others, it is detected on screening endoscopy. As the proportion of the population that receives regular health examination increases, the detection of asymptomatic PUD also appears to increase.

According to previous studies, PUD has a strong association with cigarette smoking, advanced age, former alcohol use, obesity, and specific chronic diseases. ${ }^{12}$ However, the clinical significance and pathogenic factors associated with asymptomatic PUD remain unclear to date.

Therefore, the present study aimed to investigate the prevalence of symptomatic and asymptomatic PUD in individuals receiving regular medical check-ups in Korea, and we attempted to identify risk factors for the development of symptoms in patients with PUD. 


\section{MATERIALS AND METHODS}

\section{Patients}

Individuals who underwent a health inspection at the Konkuk University Medical Center between January 2010 and January 2015 were surveyed in this retrospective study. The health inspection included upper gastrointestinal (GI) endoscopy, a serum anti-H. pylori IgG assay, and a self-report questionnaire. The following subjects were excluded from the study: subjects aged $<17$ years, subjects who underwent GI surgery, subjects with adenoma or carcinoma in the upper GI tract, subjects who did not properly answer the questionnaire, subjects using proton pump inhibitors or histamine-2 receptor antagonists, and subjects previously diagnosed with functional dyspepsia (Fig. 1). In cases in which the result of the serum $H$. pylori IgG antibody test was equivocal and other $H$. pylori tests were not performed, the patients were excluded from the study, because the presence of $H$. pylori infection could not be determined.

Subjects without PUD were categorized into the healthy control group. Among the subjects diagnosed with PUD, subjects with ulcers in the scarring stage were excluded. The remaining subjects were categorized into the symptomatic or asymptomatic PUD group according to the presence of gastroduodenal symptoms.

This study protocol was approved by the Institutional Review Board (IRB) of the Konkuk University School of Medicine (KUH1010767) and was registered in the Clinical Research Information Service (CRIS) ID KCT0001909.

\section{Questionnaires and gastroduodenal symptoms}

All examinees filled out a self-report questionnaire including questions on gastroduodenal symptoms, smoking, alcohol intake, medical history, underlying disease, and medication history. Pain, soreness, and/or burning sensation localized to the epigastric or upper abdominal area (not substernal area) for at least 3 weeks (at least once each week) during the prior 3 months were considered gastroduodenal symptoms. Drugs taken for more than 1 week during the prior 1 month were reviewed to determine the medication history, including use of NSAIDs, aspirin, proton pump inhibitors, and histamine-2 receptor blockers. Additionally, we determined subjects' age, sex, weight, and height by reviewing their medical records.

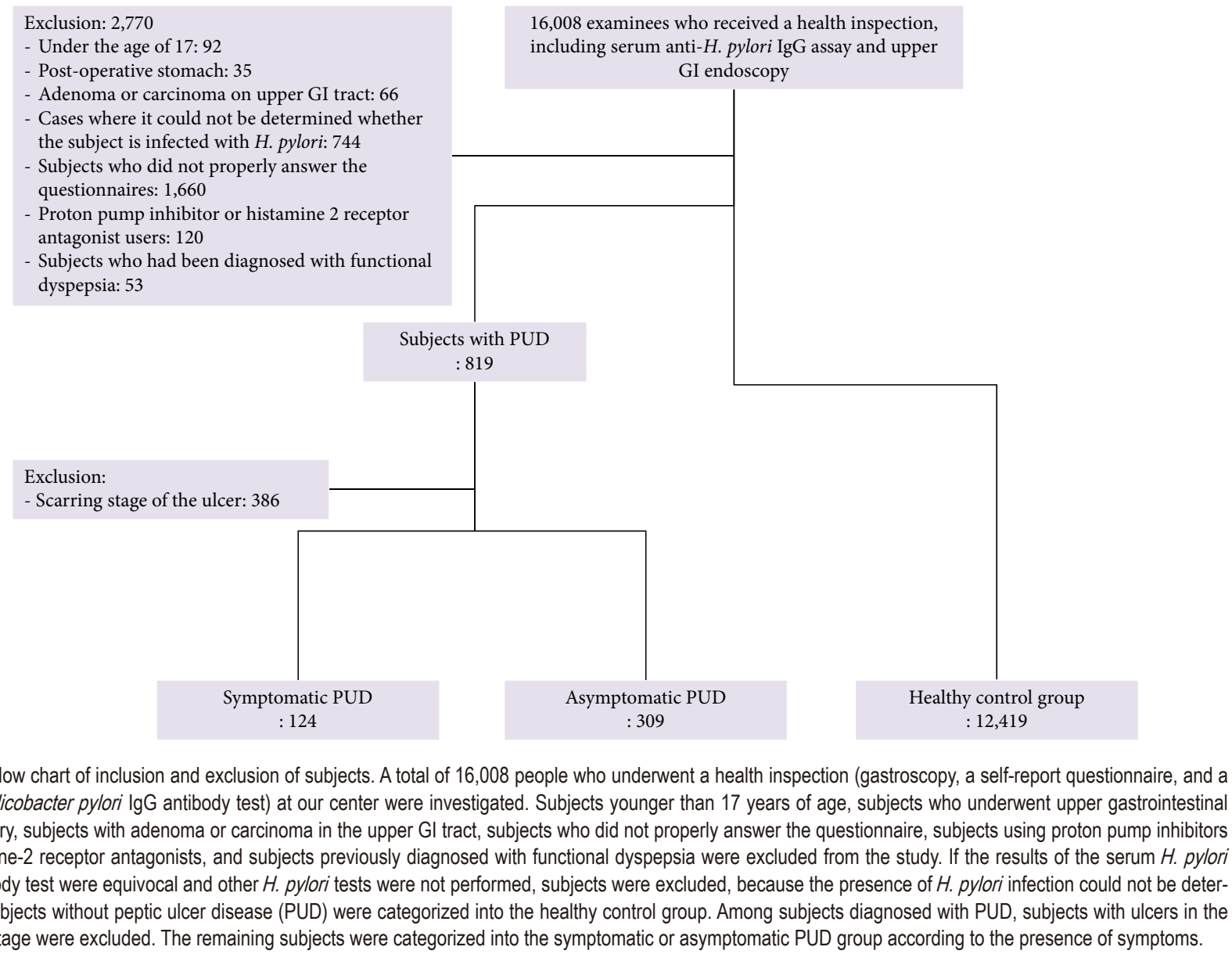

Fig. 1. Flow chart of inclusion and exclusion of subjects. A total of 16,008 people who underwent a health inspection (gastroscopy, a self-report questionnaire, and a serum Helicobacter pylori IgG antibody test) at our center were investigated. Subjects younger than 17 years of age, subjects who underwent upper gastrointestinal (GI) surgery, subjects with adenoma or carcinoma in the upper GI tract, subjects who did not properly answer the questionnaire, subjects using proton pump inhibitors or histamine-2 receptor antagonists, and subjects previously diagnosed with functional dyspepsia were excluded from the study. If the results of the serum $H$. pylori IgG antibody test were equivocal and other $H$. pylori tests were not performed, subjects were excluded, because the presence of $H$. pylori infection could not be determined. Subjects without peptic ulcer disease (PUD) were categorized into the healthy control group. Among subjects diagnosed with PUD, subjects with ulcers in the scarring stage were excluded. The remaining subjects were categorized into the symptomatic or asymptomatic PUD group according to the presence of symptoms. 


\section{Definition of PUD}

PUD was defined on the basis of the endoscopy findings as a deep mucosal defect with suspected submucosal invasion in the stomach or duodenum in the active or healing stage. Erosive esophagitis was defined as the presence of hyperemic streaks or mucosal breaks in the lower esophagus on endoscopy. The endoscopic procedure was conducted using a standard upper endoscope (GF-260, Olympus, Tokyo, Japan/EPK-I, Pentax, Tokyo, Japan). All subjects provided written consent prior to the procedure.

\section{H. pylori infection status and serum pepsinogen assay}

The H. pylori IgG antibody test was performed using enzyme-linked immunosorbent assay (ELISA) (CHORUS $H$. pylori IgG, DIESSE Diagnostica Senese, Monteriggioni, Italy) for all included subjects. The test primarily determined the presence of $H$. pylori infection. A titer of $>12 \mathrm{AU} / \mathrm{mL}$ was considered to indicate the presence of $H$. pylori infection, while a titer of $<8 \mathrm{AU} / \mathrm{mL}$ was regarded to indicate the absence of infection. Titers between $8 \mathrm{AU} / \mathrm{mL}$ and $12 \mathrm{AU} / \mathrm{mL}$ were regarded as equivocal test findings. If the results of the test were equivocal, we reviewed the patient's results for other H. pylori tests such as biopsy stained with Giemsa, urea breath test, and rapid urease test. If the result of even one of these tests was positive, the patient was considered to have $H$. pylori infection. When the $H$. pylori antibody test result was equivocal and other H. pylori tests were not conducted, the patient in question was excluded from the study. The serum pepsinogen (PG) test measured serum PG I and II concentrations by using a latex particle-enhanced turbidimetric immunoassay (HiBi

Table 1. Baseline Characteristics of All Subjects and Comparison among the Groups

\begin{tabular}{|c|c|c|c|c|c|c|}
\hline Variables & $\begin{array}{l}\text { Healthy control } \\
\text { group } \\
(A, n=12,419)\end{array}$ & $\begin{array}{c}\text { Symptomatic PUD } \\
(B, n=124)\end{array}$ & $\begin{array}{c}\text { Asymptomatic PUD } \\
(\mathrm{C}, n=309)\end{array}$ & $\begin{array}{l}p \text {-value } \\
(\text { A vs. B) }\end{array}$ & $\begin{array}{l}p \text {-value } \\
\text { (A vs. C) }\end{array}$ & $\begin{array}{l}p \text {-value } \\
\text { (B vs. C) }\end{array}$ \\
\hline Age, years ${ }^{a}$ & $47.65( \pm 12.36)$ & $50.47( \pm 11.57)$ & $49.56( \pm 12.08)$ & 0.012 & 0.007 & 0.476 \\
\hline Male sex, $n(\%)$ & $6758(54.4)$ & $94(75.8)$ & $227(73.5)$ & $<0.001$ & $<0.001$ & 0.630 \\
\hline BMI, kg/m² & $23.78( \pm 3.32)$ & $24.30( \pm 3.42)$ & $24.47( \pm 3.39)$ & 0.082 & $<0.001$ & 0.627 \\
\hline Smoking, $n(\%)$ & & & & $<0.001$ & $<0.001$ & 0.224 \\
\hline Non-smoker & $5871(54.1)$ & $28(26.2)$ & $85(31.1)$ & & & \\
\hline Past smoker & $2705(24.9)$ & $25(23.4)$ & $77(28.2)$ & & & \\
\hline Current smoker & $2282(21.0)$ & $54(50.5)$ & $111(40.7)$ & & & \\
\hline Alcohol, $n(\%)$ & & & & $<0.001$ & $<0.001$ & 0.025 \\
\hline Non-drinker & $2980(25.3)$ & $14(11.7)$ & $42(14.2)$ & & & \\
\hline Social drinker & $8208(69.6)$ & $86(71.7)$ & $230(78.0)$ & & & \\
\hline Heavy drinker ${ }^{\mathrm{b})}$ & $609(5.2)$ & $20(16.7)$ & $23(7.8)$ & & & \\
\hline H. pylori infection, $n(\%)^{c)}$ & $6865(55.3)$ & $89(71.8)$ & $218(70.6)$ & $<0.001$ & $<0.001$ & 0.816 \\
\hline Corpus atrophy, $n(\%)^{\mathrm{d})}$ & $721(13.4)$ & $5(7.4)$ & $13(8.7)$ & 0.155 & 0.111 & 0.798 \\
\hline \multicolumn{7}{|l|}{ Endoscopic findings, $n(\%)$} \\
\hline Chronic atrophic gastritis & $5646(45.5)$ & $47(37.9)$ & $119(38.5)$ & 0.103 & 0.018 & 0.914 \\
\hline Reflux esophagitis & $984(7.9)$ & $17(13.7)$ & $26(8.4)$ & 0.022 & 0.831 & 0.110 \\
\hline \multicolumn{7}{|l|}{ Underlying diseases, $n(\%)$} \\
\hline Diabetes & $799(6.4)$ & $15(12.1)$ & $24(7.8)$ & 0.017 & 0.348 & 0.193 \\
\hline \multicolumn{7}{|l|}{ Medication, $n(\%)$} \\
\hline NSAIDs & $765(6.2)$ & $15(12.1)$ & $27(8.7)$ & 0.010 & 0.073 & 0.369 \\
\hline Low dose aspirin & $845(6.8)$ & $7(5.6)$ & $28(9.1)$ & 0.722 & 0.137 & 0.253 \\
\hline
\end{tabular}

PUD, peptic ulcer disease; BMI, body mass index; NSAIDs, non-steroidal anti-inflammatory drugs.

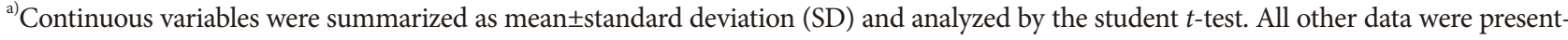
ed as number (\%) and analyzed by the chi-square test. The significant results are highlighted in bold.

${ }^{b}$ Heavy drinking was defined as consuming 15 drinks or more per week for men and 8 drinks or more per week for women.

${ }^{c} H$. pylori infection was initially identified by the results of $H$. pylori IgG antibody test. Then, for more information, the results of biopsy stained with Giemsa, urea breath test, and rapid urea test were reviewed.

${ }^{\mathrm{d})}$ Corpus atrophy was defined as a serum pepsinogen (PG) I/II ratio $<3.0$ and PG I $<70 \mathrm{ng} / \mathrm{mL}$. 
Table 2. Characteristics of the Peptic Ulcer

\begin{tabular}{lcc}
\hline Ulcer characteristics & $\begin{array}{c}\text { Symptomatic PUD } \\
(\boldsymbol{n}=\mathbf{1 2 4})\end{array}$ & $\begin{array}{c}\text { Asymptomatic PUD } \\
(\boldsymbol{n}=\mathbf{3 0 9})\end{array}$ \\
\hline Location & & \\
\hline Gastric ulcer alone & $81(65.3)$ & $215(69.6)$ \\
Duodenal ulcer alone & $30(24.2)$ & $64(20.7)$ \\
Both & $13(10.5)$ & $30(9.7)$ \\
Number & & $275(89.0)$ \\
Single ulcer & $109(87.9)$ & $34(11.0)$ \\
Multiple ulcers & $15(12.1)$ & \\
Stage & & $56(18.1)$ \\
Active stage & $39(31.5)$ & $253(81.9)$ \\
\hline Healing stage & $85(68.5)$ & 0.867 \\
\hline
\end{tabular}

PUD, peptic ulcer disease.

All data were presented as number (\%) and analyzed by the chi-square test. The significant results are highlighted in bold.

Table 3. Risk Factors for the Presence of Symptoms in PUD

\begin{tabular}{lcccccc}
\hline \multirow{2}{*}{ Variables } & \multicolumn{2}{c}{ Univariate analysis } & & & \multicolumn{2}{c}{ Multivariate analysis } \\
\cline { 2 - 3 } & OR (95\% CI) & p-value & & Adjusted OR (95\% CI) & p-value \\
Heavy drinking & $2.365(1.245-4.493)$ & 0.009 & & $2.515(1.315-4.812)$ & 0.005 \\
Active stage ulcer & $2.073(1.287-3.340)$ & 0.003 & & $2.143(1.323-3.472)$ & 0.002 \\
\hline
\end{tabular}

PUD, peptic ulcer disease; OR, odds ratio; CI, confidence interval.

Univariate and multivariate logistic regression analyses were performed and the significant results are highlighted in bold.

Co., Anyang, Korea). Corpus atrophy was defined as a serum PG I/II ratio $<3.0$ and PG I levels $<70 \mathrm{ng} / \mathrm{mL}$, as described previously. $^{13}$

\section{Statistical analysis}

Continuous variables were analyzed using Student's $t$-test, and their values are presented as mean \pm standard deviation (SD). Categorical variables were analyzed using the $\chi^{2}$ test, and their values are presented as number (\%). Logistic regression analyses were performed to estimate the odds ratio (OR) and 95\% confidence interval (CI) for factors associated with the existence of PUD, and for factors associated with the presence of symptoms among patients with PUD. All analyses were conducted using SPSS version 19.0 (SPSS Inc., Chicago, IL, USA), and a $p$-value $<0.05$ was considered statistically significant.

\section{RESULTS}

\section{Subjects characteristics}

A total of 12,852 subjects were enrolled in this study, of whom 7,079 (55.1\%) were men. Among the total subjects, $12,419(96.6 \%)$ were assigned to the healthy control group,
124 to the symptomatic PUD group (1.0\%), and 309 (2.4\%) to the asymptomatic PUD group (Table 1). The mean age and body mass index (BMI) of subjects in the healthy control group were $47.65 \pm 12.36$ years and $23.78 \pm 3.32 \mathrm{~kg} / \mathrm{m}^{2}$, those of subjects in the symptomatic PUD group were $50.47 \pm 11.57$ years and $24.30 \pm 3.42 \mathrm{~kg} / \mathrm{m}^{2}$, and those of subjects in the asymptomatic PUD group were $49.56 \pm 12.08$ years and $24.47 \pm 3.39 \mathrm{~kg} / \mathrm{m}^{2}$.

\section{Risk factors for the presence of symptoms in pa- tients with PUD}

Subjects with symptomatic PUD had higher alcohol consumption than those with asymptomatic PUD $(p=0.025)$ (Table 1). Furthermore, the presence of active-stage ulcers was more common in subjects with symptomatic PUD than in subjects with asymptomatic PUD $(p=0.003)$ (Table 2). Between the symptomatic and asymptomatic PUD groups, there were no significant differences in age, sex, BMI, smoking, $H$. pylori infection, use of NSAIDs, ulcer location, or ulcer number. Multivariate analysis revealed that heavy drinking (OR, 2.515; 95\% CI, 1.315-4.812; $p=0.005)$ and active-stage ulcer (OR, 2.143; 95\% CI, 1.323-3.472; $p=0.002$ ) were independent risk factors for the presence of symptoms in PUD (Table 3). 
Table 4. Risk Factors for Symptomatic and Asymptomatic PUD

\begin{tabular}{|c|c|c|c|c|c|}
\hline & \multirow{2}{*}{ Variables } & \multicolumn{2}{|c|}{ Univariate analysis } & \multicolumn{2}{|c|}{ Multivariate analysis } \\
\hline & & OR $(95 \% \mathrm{CI})$ & $p$-value & Adjusted OR (95\% CI) & $p$-value \\
\hline \multirow[t]{8}{*}{ Symptomatic PUD } & Age & 1.018 (1.004-1.033) & 0.012 & 1.025 (1.008-1.043) & 0.004 \\
\hline & Male sex & $2.625(1.738-3.965)$ & $<0.001$ & $1.415(0.861-2.326)$ & 0.171 \\
\hline & Current smoker & $3.829(2.614-5.609)$ & $<0.001$ & $3.468(2.235-5.380)$ & $<0.001$ \\
\hline & Heavy drinker & $3.674(2.258-5.979)$ & $<0.001$ & $1.643(0.912-2.960)$ & 0.098 \\
\hline & H. pylori infection & $2.057(1.389-3.047)$ & $<0.001$ & $1.899(1.237-2.917)$ & 0.003 \\
\hline & Reflux esophagitis & $1.846(1.102-3.093)$ & 0.020 & $1.539(0.870-2.722)$ & 0.138 \\
\hline & Diabetes & $2.001(1.161-3.450)$ & 0.013 & $1.336(0.723-2.470)$ & 0.355 \\
\hline & NSAIDs & $2.096(1.216-3.614)$ & 0.008 & $1.905(1.031-3.519)$ & 0.040 \\
\hline \multirow[t]{7}{*}{ Asymptomatic PUD } & Age & $1.012(1.003-1.022)$ & 0.007 & $1.024(1.013-1.035)$ & $<0.001$ \\
\hline & Male sex & $2.319(1.797-2.992)$ & $<0.001$ & $1.871(1.362-2.569)$ & $<0.001$ \\
\hline & BMI & $1.062(1.028-1.096)$ & $<0.001$ & $1.023(0.984-1.063)$ & 0.258 \\
\hline & Current smoker & $2.575(2.014-3.293)$ & $<0.001$ & $2.134(1.617-2.818)$ & $<0.001$ \\
\hline & Heavy drinker & $1.553(1.007-2.396)$ & 0.046 & $1.069(0.671-1.702)$ & 0.779 \\
\hline & H. pylori infection & $1.938(1.514-2.482)$ & $<0.001$ & $2.186(1.657-2.883)$ & $<0.001$ \\
\hline & No atrophic gastritis & $1.331(1.056-1.678)$ & 0.016 & $1.854(1.419-2.424)$ & $<0.001$ \\
\hline
\end{tabular}

PUD, peptic ulcer disease; OR, odds ratio; CI, confidence interval; NSAIDs, non-steroidal anti-inflammatory drugs; BMI, body mass index. Univariate and multivariate logistic regression analyses were performed and the significant results are highlighted in bold.

\section{Comparison of PUD groups with healthy control group}

Subjects with symptomatic PUD were older $(p=0.012)$, predominantly male $(p<0.001)$, more likely to be smokers $(p<0.001)$, and had higher alcohol consumption $(p<0.001)$ than the healthy control subjects in addition, this group had a higher number of subjects with $H$. pylori infection $(p<0.001)$, reflux esophagitis $(p=0.022)$, diabetes $(p=0.017)$, and NSAID use $(p=0.010)$ than the healthy control group (Table 1). In contrast, subjects with asymptomatic PUD were older $(p=0.007)$, predominantly male $(p<0.001)$, had a higher BMI $(p<0.001)$, were more likely to be smokers $(p<0.001)$, and had higher alcohol consumption $(\mathrm{p}<0.001)$ than the healthy control subjects; this group also had a higher number of subjects with $H$. pylori infection $(p<0.001)$ and a lower number of subjects with chronic atrophic gastritis $(p=0.018)$ than the healthy control group.

Using multivariate logistic regression analysis, old age (OR, 1.025 ; 95\% CI, 1.008-1.043; $p=0.004$ ), current smoking (OR, 3.468; 95\% CI, 2.235-5.380; $p<0.001$ ), H. pylori infection (OR, $1.899 ; 95 \%$ CI $1.23-2.917 ; p=0.003$ ), and use of NSAIDs (OR, $1.905 ; 95 \%$ CI, 1.031-3.519; $p=0.040$ ) were found to be independent risk factors for symptomatic PUD (Table 4). Meanwhile, old age (OR, 1.024; 95\% CI, 1.013-1.035; $p<0.001$ ), male sex (OR, 1.871; 95\% CI, 1.362-2.569; $p<0.001$ ), current smok- ing (OR, 2.134; 95\% CI, 1.617-2.818; $p<0.001$ ), H. pylori infection (OR, 2.186; 95\% CI, 1.657-2.883; $p<0.001$ ), and absence of atrophic gastritis (OR, 1.854; 95\% CI, 1.419-2.424; $p<0.001$ ) were independent risk factors for asymptomatic PUD.

\section{Presence of symptoms in the healthy control group}

In the healthy control group, 1152 (9.3\%) subjects had gastroduodenal symptoms (Supplementary Table 1). These subjects were younger $(p<0.001)$, predominantly female $(p<0.001)$, more likely to be smokers $(p<0.001)$, and had higher alcohol consumption $(p<0.001)$ than subjects in the healthy group without gastroduodenal symptoms; in addition, as compared to the healthy group without gastroduodenal symptoms, the healthy group with gastroduodenal symptoms included a lower number of subjects with $H$. pylori infections $(p<0.001)$, corpus atrophy $(p<0.001)$, and atrophic gastritis $(p=0.002)$, as well as fewer low-dose aspirin users $(p=0.033)$ and a higher number of subjects using NSAIDs $(p<0.001)$.

\section{DISCUSSION}

In the present study, we found that active-stage ulcers triggered more symptoms than healing-stage ulcers, and the presence of active-stage ulcers was an independent risk 
factor for the presence of symptoms in PUD. Gastroduodenal symptoms can develop more often in the active stage of ulcers, because the gastric mucosa is damaged to the highest extent in this stage. In addition, ulcer location and number were not related to symptoms. It is speculated that patients with healing-stage ulcers show symptoms less frequently, even if they have several peptic ulcers.

In this study, heavy drinking was the most powerful risk factor for the presence of symptoms in patients with PUD. Excessive alcohol consumption by patients with PUD may be related to gastroduodenal symptoms. However, heavy drinking can induce symptoms, with or without PUD. ${ }^{14}$ In our study, heavy drinking was related to gastroduodenal symptoms not only in subjects with PUD but also in healthy individuals. Therefore, further studies will be required to determine whether heavy drinking is responsible for the development of symptoms in PUD.

The use of NSAIDs was a risk factor for symptomatic PUD but not for asymptomatic PUD in the present study, and $H$. pylori infection was a risk factor for both symptomatic and asymptomatic PUD. These findings indicate that NSAID-induced PUD may lead to symptoms more often than $H$. pylo$r i$-associated PUD. ${ }^{15}$ According to the current study, the use of NSAIDs was related to gastroduodenal symptoms in the healthy control group as well. Therefore, NSAIDs can cause gastroduodenal symptoms, regardless of the presence of PUD, a finding consistent with previous study results. ${ }^{16}$

In the present study, H. pylori infection itself was a powerful risk factor for PUD in Korean adults, regardless of the presence of gastroduodenal symptoms. However, the absence of chronic atrophic gastritis was associated with asymptomatic PUD. This result suggests that ulcer-related symptoms may be reduced in patients with advanced stages of $H$. pylori infection. As the infection persists over a long period, atrophic changes of the gastric and duodenal mucosa become more severe. ${ }^{17}$ However, chronic inflammation of the mucosa may desensitize patients to the mucosal damage and reduce their symptoms. ${ }^{18}$ This inverse relationship between gastroduodenal symptoms and chronic atrophic gastritis was also observed in the healthy control group (Supplementary Table 1).

Old age and smoking are well-known risk factors for PUD; these were also found to be risk factors for PUD in the present study, regardless of the presence of symptoms. ${ }^{19}$ On the other hand, male sex was a risk factor for asymptomatic PUD, but not for symptomatic PUD in our study. Previous studies revealed that female sex is a common predisposing factor for symptoms of functional GI disorders. ${ }^{20-22}$ Because men are less sensitive to GI symptoms, ulcer-related symptoms may be less obvious in men than in women.

Despite our important findings, the present study has some limitations. First, it was nearly impossible to determine the causal relationship between gastroduodenal symptoms and PUD. In this study, 9.3\% of subjects in the healthy control group had gastroduodenal symptoms. Although individuals diagnosed with functional dyspepsia were excluded from the study based on survey responses, some subjects included in the study may have had functional GI disorders. Similarly, some symptoms may have been triggered by minor injury to the gastroduodenal wall. In other words, symptoms of some subjects with symptomatic PUD may not be caused by the peptic ulcers. Second, some values were missing in our data, which included the smoking history of 1,614 subjects, the alcohol-consumption history of 640 subjects, and the BMI of 23 subjects; additionally, the serum PG of 7,261 subjects was not measured. Finally, the relationship between atrophic change of the gastric mucosa and upper GI symptoms remains a highly contentious issue. ${ }^{18,23,24} \mathrm{We}$ believe that severe atrophic change of the gastric mucosa may reduce a patient's gastroduodenal symptoms, but this hypothesis has not been completely reliable thus far. In the present study, atrophic gastritis diagnosed by endoscopy was inversely related to symptoms. However, PG assay results did not show any relationship to symptoms.

In conclusion, in Korea, a country in which $H$. pylori infection is endemic, the incidence of symptomatic and asymptomatic PUD found on screening endoscopy was 1.0\% and $2.4 \%$, respectively. Old age, current smoking, and $H$. pylori infection were independent risk factors for symptomatic and asymptomatic PUD. Furthermore, use of NSAIDs was an independent risk factor only for symptomatic PUD. These results suggest that NSAID-induced PUD may lead to symptoms more often than $H$. pylori-associated PUD. Heavy drinking and active-stage ulcers were independent risk factors for the presence of gastroduodenal symptoms in patients with PUD. Active-stage ulcers may trigger more symptoms than healing-stage ulcers, regardless of their location and number.

Conflicts of Interest

The authors have no financial conflicts of interest.

\section{Supplementary Material}

Supplementary material is available from https://doi.org/10.5946/ ce.2016.129 or via http://e-ce.org/.

\section{REFERENCES}

1. Milosavljevic T, Kostić-Milosavljević M, Jovanović I, Krstić M. Complications of peptic ulcer disease. Dig Dis 2011;29:491-493.

2. Al Dhahab H, McNabb-Baltar J, Al-Taweel T, Barkun A. State-of-the-art management of acute bleeding peptic ulcer disease. Saudi J Gastroen- 
terol 2013;19:195-204.

3. Wolfe MM, Lichtenstein DR, Singh G. Gastrointestinal toxicity of nonsteroidal antiinflammatory drugs. N Engl J Med 1999;340:1888-1899.

4. Hopkins RJ, Girardi LS, Turney EA. Relationship between Helicobacter pylori eradication and reduced duodenal and gastric ulcer recurrence: a review. Gastroenterology 1996;110:1244-1252.

5. Atherton JC, Cao P, Peek RM Jr, Tummuru MK, Blaser MJ, Cover TL. Mosaicism in vacuolating cytotoxin alleles of Helicobacter pylori. Association of specific vacA types with cytotoxin production and peptic ulceration. J Biol Chem 1995;270:17771-17777.

6. Hunt RH, Yuan Y. Acid-NSAID/aspirin interaction in peptic ulcer disease. Dig Dis 2011;29:465-468.

7. Yamaoka Y, Ojo O, Fujimoto S, et al. Helicobacter pylori outer membrane proteins and gastroduodenal disease. Gut 2006;55:775-781.

8. Potamitis GS, Axon AT. Helicobacter pylori and Nonmalignant Diseases. Helicobacter 2015;20 Suppl 1:26-29.

9. Thorat MA, Cuzick J. Prophylactic use of aspirin: systematic review of harms and approaches to mitigation in the general population. Eur J Epidemiol 2015;30:5-18.

10. Sasaki H, Nagahara A, Hojo M, et al. Ten-year trend of the cumulative Helicobacter pylori eradication rate for the 'Japanese eradication strategy'. Digestion 2013;88:272-278.

11. Kim JH, Kim HY, Kim NY, et al. Seroepidemiological study of Helicobacter pylori infection in asymptomatic people in South Korea. J Gastroenterol Hepatol 2001;16:969-975.

12. Lau JY, Sung J, Hill C, Henderson C, Howden CW, Metz DC. Systematic review of the epidemiology of complicated peptic ulcer disease: incidence, recurrence, risk factors and mortality. Digestion 2011;84:102-113.

13. Choi HS, Lee SY, Kim JH, et al. Combining the serum pepsinogen level and Helicobacter pylori antibody test for predicting the histology of gastric neoplasm. J Dig Dis 2014;15:293-298.

14. Halder SL, Locke GR 3rd, Schleck CD, Zinsmeister AR, Talley NJ. Influ- ence of alcohol consumption on IBS and dyspepsia. Neurogastroenterol Motil 2006;18:1001-1008.

15. Kim HM, Cho JH, Choi JY, et al. NSAID is inversely associated with asymptomatic gastric ulcer: local health examination data from the Korean National Health Insurance Corporation. Scand J Gastroenterol 2013;48:1371-1376.

16. Yap PR, Goh KL. Non-steroidal anti-inflammatory drugs (NSAIDs) induced dyspepsia. Curr Pharm Des 2015;21:5073-5081.

17. D'Elios MM, Andersen LP. Inflammation, immunity, and vaccines for Helicobacter pylori. Helicobacter 2009;14 Suppl 1:21-28.

18. Tahara T, Arisawa T, Shibata T, et al. Association of endoscopic appearances with dyspeptic symptoms. J Gastroenterol 2008;43:208-215.

19. Li LF, Chan RL, Lu L, et al. Cigarette smoking and gastrointestinal diseases: the causal relationship and underlying molecular mechanisms (review). Int J Mol Med 2014;34:372-380.

20. Lee SP, Sung IK, Kim JH, Lee SY, Park HS, Shim CS. The effect of emotional stress and depression on the prevalence of digestive diseases. J Neurogastroenterol Motil 2015;21:273-282.

21. Ford AC, Bercik P, Morgan DG, Bolino C, Pintos-Sanchez MI, Moayyedi P. Characteristics of functional bowel disorder patients: a cross-sectional survey using the Rome III criteria. Aliment Pharmacol Ther 2014;39:312-321.

22. Oshima T, Miwa H. Epidemiology of functional gastrointestinal disorders in Japan and in the world. J Neurogastroenterol Motil 2015;21:320329.

23. Bode G, Brenner H, Adler G, Rothenbacher D. Dyspeptic symptoms in middle-aged to old adults: the role of Helicobacter pylori infection, and various demographic and lifestyle factors. J Intern Med 2002;252:41-47.

24. Stone MA, Barnett DB, Mayberry JF. Lack of correlation between self-reported symptoms of dyspepsia and infection with Helicobacter pylori, in a general population sample. Eur J Gastroenterol Hepatol 1998;10:301-304 